\title{
Posterior surgery assisted by three dimensional printing technology in the treatment of sacroiliac joint tuberculosis
}

Xinhua Yin

Xi'an Red Cross Hospital

Liang Yan

Xi'an Red Cross Hospital

Ding Jun Hao

Xi'an Red Cross Hospital

Xiao Bin Yang

Xi'an Red Cross Hospital

Zhongkai Liu ( $\nabla$ spine2020@163.com )

department of spine surgery, HongHui Hospital

Research article

Keywords: Three dimensional printing technology, Sacroiliac joint tuberculosis, Posterior sacroiliac screws fixation

Posted Date: March 17th, 2020

DOl: https://doi.org/10.21203/rs.3.rs-17555/v1

License: (c) (i) This work is licensed under a Creative Commons Attribution 4.0 International License. Read Full License 


\section{Abstract}

\section{Background}

Three-dimensional (3D) printing is becoming increasingly important in spinal surgery, and few literatures have reported the surgical management for sacroiliac joint tuberculosis assisted by three dimensional printing technology. Purpose The study aim to assess the efficacy and feasibility of surgical treatment in sacroiliac joint tuberculosis (SJT) by posterior sacroiliac screws fixation, debridement and fusion, which assisted by three dimensional printing technology.

\section{Method}

Twenty-six patients with sacroiliac joint tuberculosis treated by surgery in our department between January 2008 and December 2015 . All the patients were divided into two group base on three dimensional printing technology: 11 cases in Group A underwent posterior debridement, fusion and sacroiliac screws fixation, and 15 cases in Group B underwent posterior debridement, bone graft, sacroiliac screws fixation, and three dimensional printing technology. Clinical and radiographic results for the two groups were analyzed and compared.

Results

All patients were followed up with an average $33.8 \pm 7.9$ months (24-48 months). The sacroiliac joint tuberculosis was completely cured and the grafted bones were fused in all 26 patients. No injured nerve or other severe complications occurred in both groups. Group B achieved much better results in time of operation, intra-operative blood loss, and number of intra-operative fluoroscopy than group A $(p<0.05)$. There were no significant differences between the two groups in ESR, fusion time, and VAS score in the last follow-up ( $p>0.05)$.

\section{Conclusions}

The three dimensional printing technology can achieve the better effect in the treatment of sacroiliac joint tuberculosis. Posterior surgery assisted by three dimensional printing technology in the treatment of sacroiliac joint tuberculosis has the characteristics of shorter operative time, less bleeding, and less number of intra-operative fluoroscopy.

\section{Introduction}

Tuberculosis(TB)is the ninth leading cause of death worldwide. According to a WHO report in 2019[1], there were an estimated 1.5 million TB deaths. China has the second highest burden of tuberculosis in the world, registering $17 \%$ of all global cases [2]. Skeletal tuberculosis accounts for $3-5 \%$ of all tuberculosis, of which approximately $2-10 \%$ occurs at the sacroiliac joint [3-6]. The treatment principles of SJT are basically derived from the experience in treating spinal tuberculosis. Surgeries are being performed for SJT such as abscess drainage, neurological deficit, biopsy, drug resistance and instability 
[7-8]. With the advent of three dimensional printing technology and modern spinal surgery, accurate and individualized surgery is possible in treatment of spine disorder. The purpose of our study is to use 3D printing technology for preoperative planning, surgical simulation, intraoperative guidance in SJT. This study aim to evaluate the clinical efficacy of the surgery assisted by 3D printing technology in treatment of SJT.

\section{Materials And Methods}

\section{Basic information}

A total of 26 patients with SJT received surgical treatment in our hospital between January 2008 and December 2015. The mean interval between symptom onset and clinical diagnosis was $5.7 \pm 3.1$ months (range, 2-12 months). All patients had symptoms of low back pain, buttock, weight loss, low fever and fatigue, 20 of whom complain of difficulty walking. Patrick's test and pelvic compression tests were positive in the affected side. Ranges of motion at the affected hips was limited in all patients. All patients affecting a single joint. The diagnosis of SJT was based on clinical symptoms, imageological examination (spinal $\mathrm{X}$-ray films, computed tomography, magnetic resonance imaging) and laboratory data (erythrocyte sedimentation rate, liver and renal function tests and so on). The patients were divided into two groups according to whether 3D printing technology is used. Group A, 11 cases receive posterior debridement, fusion and sacroiliac screws fixation, and 15 cases receive posterior debridement, bone graft, sacroiliac screws fixation, and three dimensional printing technology in Group B. Clinical outcome was assessed preoperatively and at the last follow-up visit using Visual analog scale (VAS) questionnaire. The patients were treated with four drugs regime:Isoniazid, Rifampicin, Ethambutol and Pyrazinamide(HREZ). The chemotherapy was preoperatively carried out for 7 to 14 days. The enhancement of nutrition for correction of anemia and hypoproteinemia in all patients. There were no significant differences between the two groups in age, sex ratio. Patients' general information is summarized in Table 1.

Table 1

Clinical data on the patients

\begin{tabular}{|c|c|c|c|}
\hline & \multicolumn{2}{|c|}{ Gender } & \multirow[t]{2}{*}{ Age(year) } \\
\hline & Male & Female & \\
\hline Group A & 7 & 4 & $34.7 \pm 13.5$ \\
\hline Group B & 9 & 6 & $32.9 \pm 10.0$ \\
\hline T value & \multicolumn{2}{|c|}{$x^{2}=0.034$} & $t=0.389$ \\
\hline$P$ value & \multicolumn{2}{|c|}{0.854} & 0.701 \\
\hline
\end{tabular}




\section{Surgery Simulation}

In the 3D printed models, it is benefit to design operative plan, choose the optimization approach, and teach the processes of operations and anatomy. In addition, the doctors were able to choose ideal screws types, location, and orientation before operation.

\section{Operation Procedure}

Surgery was performed under general anaesthesia and the patients were kept in prone position. The sacroiliac joint were exposed by $8-10 \mathrm{~cm}$ arc incision. A bone window was chiseled at the corresponding position of the lesion. Next, we used a flush tube to drain abscesses. Including sequestra and cold abscesses were completely removed by curettes through the healthy bleeding bone. Bone defect space was packed with autogenous bone which was acquired from the healthy iliac crest. Finally, the sterile physiologic saline, $0.2 \mathrm{~g}$ isoniazid and $1.0 \mathrm{~g}$ intramuscular streptomycin were irrigated by in the surgical area; multi-functional drainage tube were performed. The material debrided was sent for culture and histopathologic examination.

\section{Post-operative Management}

The drain was usually pulled out when drainage flow was less than $50 \mathrm{ml} /$ day. Patients remained in bed for 3 to 5 days post-operatively, then patients were allowed to ambulate and rehabilitation are initiated. All patients received anti-tuberculosis therapy with HREZ for the next 3 months, and then with HRE treatment for another 9-15 months. The braces were used for a minimum period of 3 months post-operatively. The intraoperative blood loss, radiation times, operation time, length of stay, VAS (Visual analogue score) and bone fusion time of both groups were measured. The laboratory examination (hepatic and kidney function, blood test, erythrocyte sedimentation rate) and imageological examination were carried out post-operatively at $3,6,12,18,24$ months and once a year. The data was analyzed using SPSS 20 . The pre and postoperative data were compared with paired Student's $t$ test, and comparisons between groups using chi-squared test and Student's t test. Differences were considered significant at $p<0.05$. The results are recorded as mean standard deviation (SD).

\section{Results}

There was no SJT recurrence occurred in the series. All patients had significant improvement in clinical symptoms postoperatively. The duration of follow-up ranged from 24 to 48 months (mean $33.8 \pm 7.9$ ). All patients achieved bone fusion. Group B achieved much better results in time of operation, the times of radiation, and intra-operative blood loss than group $A(P<0.05$, Table 2$)$. Whereas, there were no significant differences between the two groups in VAS score, and the time of bone fusion in the last follow-up ( $P>0.05$, Table 2). No severe complications occurred in both groups. The screws loosening was observed in one patient (group A), because of re-adjust of screw position during the operation. The 
screws removed in second operation when the patients had good bone graft fusion. In group A, one patients reported harvest site pain postoperatively, while one patient in Group B. They all treated with physical therapy at final follow up. The representative case was attached as Fig. 1.

Table 2

Comparison of results between two groups

\begin{tabular}{|llll|}
\hline & Group A(n=11) & Group B ( $\mathbf{n = 1 5 )}$ & p value \\
\hline Blood loss (ml) & $688.2 \pm 159.0$ & $490.6 \pm 131.9$ & 0.012 \\
\hline Operative time(mins) & $266.4 \pm 49.1$ & $216.7 \pm 63.7$ & 0.038 \\
\hline Hospital stay (days) & $8.8 \pm 1.4$ & $8.9 \pm 1.6$ & 0.829 \\
\hline Fusion time (mons) & $7.9 \pm 1.4$ & $7.8 \pm 1.3$ & 0.772 \\
\hline Radiation times & $7.5 \pm 1.3$ & $4.8 \pm 1.0$ & 0.001 \\
\hline VAS & & & \\
\hline Preoperatively & $6.1 \pm 0.8$ & $6.0 \pm 1.0$ & 0.146 \\
\hline Final follow-up & $1.5 \pm 0.5$ & $1.7 \pm 0.4$ & 0.286 \\
\hline
\end{tabular}

\section{Discussion}

Sacroiliac joint tuberculosis is relatively uncommon and it is reported by case reports in the Englishlanguage literature. The previous study reports that SJB make up approximately $2-10 \%$ of spine tuberculosis, which tends to occur in adolescents and young adults. In the present series, the mean age of patients with SJT was 32 years which was consistent with the findings of Kim et al [9] and Ramlakan et al [10]. More often than not, the blood supply to the sacroiliac joint decline after the third decade of life. It is inferred that the change of sacroiliac joint blood supply was the key mechanism [11]. The mean interval from symptom onset to diagnosis is reported to be 16 to 19 months [12], but in our series, he mean interval between, but in our series was $5.7 \pm 3.1$ months(2-12months). We hypothesized that the improvement of diagnostic technique maybe result from the clinical difference. Whereas, the diagnosis of sacroiliac joint tuberculosis is challenging and often misleading, either patients lack of specific symptoms in the early phase, or it easily to ignore sacroiliac joint because patients are examined in the supine position. Clinically, the most frequent symptoms is buttock or lumbar pain in SJT patients. The incidence of the fever is variable, in the present study, eight patients $(30.7 \%)$ had fever. Our study show elevated ESR in all patients. Similarly, The result shown by Zhu et al [13]. and Kim et al [9]. The previous research showed that magnetic resonance imaging is high sensitive modality in the early diagnosis of SJT, associated capsular distention, cartilage and osseous erosions. In our series, MRI was done in all patients preoperatively. The diagnosis of SJT should be suspected in the presence of certain clinical and radiologic findings, laboratory data, and drug response in most patients, however, the histopathology is the gold standard for diagnosis of SJT. 
The basic principles of treatment for SJT that we referred to the experience in treating spinal tuberculosis [14-16]. Treatment strategies for sacroiliac joint tuberculosis include both conservative and operative treatments. Surgery is frequently imperative, if patients suffer from failure of conservative treatment, or progression of joint instability or neurological dysfunction, or persistent pain. However, surgical treatment of the sacroiliac joint infection is quite challenging because of the complex anatomy of the sacroiliac joint junction. As a result of the advantages of 3D printing, the new technology has been extensively used in orthopaedics [17]. As we know, 3D printing technology provide shorter, less invasive, more precise, and more reliable surgeries [18]. It provides 3D models for surgical simulation and specific implant selection. Here we describe posterior surgical treatment of SJT assisted by three dimensional printing technology. The approach creates enough operating room allowing operation under direct visualisation for radical debridement without injuring lumbosacral plexus and blood vessel. Additionally, Broner et al. [19] and Weisz [20] have confirmed that spinal stability contribute to suppress spinal infection. The stable internal environment could decrease spinal tuberculosis relapse [21]. In our series, spinal stability was unaffected, and all patients got bony fusion. And all patients recovered from tuberculosis. We compared the traditional surgery with 3D printing technology surgery in the treatment of SJT. It showed that the 3D printing group achieved much better results in time of operation, intra-operative blood loss, and number of intra-operative fluoroscopy than group $A(P<0.05)$. There were no significant differences between the two groups in fusion time and VAS score in the last follow-up.

From our experience, the treatment of SJT should focus on the following points. Firstly, the 3D printing technology were analyzed for a better preoperative planning. Secondly, the medical dispute between doctors and patients mainly manifests in the different cognition of diseases and the understanding of surgery program. The good communication between doctor and patient is benefical to improve the clinical outcome have reported by Cockburn et al [22] and Dyche et al [23]. In our cohorts, 3D printing technology could help to better understand the anatomy and pathology, improve patient's understanding and reduce the medical dispute. Thirdly, we applied a simulated virtual surgery on a 3D-printed model not only to choose ideal screw length and the direction but also to design the range of debridement prior to the actual surgery. Fourthly, pre-operative immobilization and nutrition support need to be emphasized. Finally, but not insignificantly, despite advances in surgical techniques, chemotherapy remains the cornerstone of SJTB treatment.

\section{Conclusion}

This study suggests that posterior surgery assisted by 3D printing technology in treatment of sacroiliac joint tuberculosis can be an effective and feasible treatment method. It have the characteristics of better understand the disease, better preoperative planning, shorter operative time, less bleeding, and less number of intra-operative fluoroscopy.

\section{Abbreviations}


3D:Three-dimensional, SJT: sacroiliac joint tuberculosis, TB: Tuberculosis, HREZ: Isoniazid, Rifampicin, Ethambutol and Pyrazinamid, VAS: Visual analogue score

\section{Declarations}

\section{Ethics approval and consent to participate}

This study is a retrospective clinical study and has been approved by the HongHui Hospital Ethics Committee. All patients had signed the consent form.

\section{Consent for publication}

Not applicable.

\section{Availability of data and material}

The datasets used and/or analyzed during the current study are available from the corresponding author on reasonable request.

\section{Declarations}

The authors declare that they have no conflicts of interest.

\section{Funding}

This publication was funded by Natural Science Foundation of the Department of Science and Technology of ShanXi province (2019JM-200)

\section{Authors' contributions}

ZKL contributed to the study design. DJH performed the surgery, LY collected the data. BXY analyzed the data. XHY wrote the manuscript. All authors read and approved the final manuscript.

Zhong Kai Liu and Xiao Bin Yang contributed equally to this work

\section{Acknowledgements}

Not applicable.

\section{References}

1. World Health Organization. (2019). Global tuberculosis report 2018. 2018. Geneva: World Health.

2. Wells WA, Konduri N, Chen C, Lee D, Ignatius HR, Gardiner E, Schwalbe NR (2010) Tuberculosis regimen change in high-burden countries. Int J Tuberc Lung Dis 14(12):1538-1547

3. Garg RK, Somvanshi DS. Spinal tuberculosis: a review J. J Spinal cCord Med. 2011;34(5):440-54. 
4. Gelal F, Sabah D, Dogan R, Avci A. Multifocal skeletal tuberculosis involving the lumbar spine and a sacroiliac joint: MR imaging findings[J]. Diagn Interv Radiol. 2006;12(3):139-41.

5. Pouchot J, Vinceneux P, Barge J, Boussougant Y, Grossin M, Pierre J, et al. Tuberculosis, of the sacroiliac joint: clinical features, outcome, and evaluation of closed needle biopsy in 11 consecutive cases[J]. Am J Med. 1988;84(3):622-8.

6. Goldberg J, Kovarsky J (1983) Tuberculous sacroiliitis. South Med J 76(9):1175-1176

7. Yin X H, He B R, Liu Z K, et al. The clinical outcomes and surgical strategy for cervical spine tuberculosis: A retrospective study in 78 cases.[J]. Medicine, 2018, 97(27):e11401.

8. Wang L J, Zhang H Q, Tang M X, et al. Comparison of Three Surgical Approaches for Thoracic Spinal Tuberculosis in Adult: Minimum 5-Year Follow Up[J]. Spine, 2017, 42(11):808-817.

9. Kim N H, Lee H M, Yoo J D, et al. Sacroiliac joint tuberculosis. Classification and treatment.[J]. Clinical Orthopaedics \& Related Research, 1999, 358(358):215-222.

10. Ramlakan R J, Govender S. Sacroiliac joint tuberculosis.[J]. International Orthopaedics, 2007, 31(1):121-124.

11. Zimmermann B, Mikolich DJ, Lally EV. Septic sacroiliitis. Semin Arthritis Rheum. 1996;26:592-604.

12. Moore SL, Rafii M (2001) Imaging of musculoskeletal and spinal tuberculosis. Radiol Clin North Am 39:329-342

13. Zhu G , Jiang L Y , Yi Z , et al. Sacroiliac joint tuberculosis: surgical management by posterior openwindow focal debridement and joint fusion[J]. BMC Musculoskeletal Disorders, 2017, 18(1):504.

14. Shen J, Zheng Q, Wang Y, et al. One-stage combined anterior-posterior surgery for thoracic and lumbar spinal tuberculosis[J]. The journal of spinal cord medicine, 2019: 1-8.

15. Huang Z, Liu J, Ma K. Posterior versus Anterior Approach Surgery for Thoracolumbar Spinal Tuberculosis[J]. Journal of the College of Physicians and Surgeons Pakistan, 2019, 29(2): 187-188.

16. Li Z W, Li Z Q, Tang B M, et al. Efficacy of One-Stage Posterior Debridement and Bone Grafting with Internal Fixation in the Treatment of Monosegmental Thoracolumbar Tuberculosis[J]. World neurosurgery, 2019, 121: e843-e851.

17. Li Z, Li Z, Xu R, et al. Three-dimensional printing models improve understanding of spinal fracture-A randomized controlled study in China[J]. Sci Rep, 2015, 5:11570.

18. Zeng $C$, Xing $W, W u Z$, et al. A combination of three-dimensional printing and computer-assisted virtual surgical procedure for preoperative planning of acetabular fracture reduction[J]. Injuryinternational Journal of the Care of the Injured, 2016, 47(10):2223-2227.

19. Broner FA, Garland DE, Zigler JE (1996) Spinal infections in the immunocompromised host. Orthop Clin N Am 27(1):37-46

20. Weisz RD, Errico TJ (2000) Spinal infections. Diagnosis and treatment. Bull Hosp Jt Dis 59(1):40-46

21. Altman GT, Altman DT, Frankovitch KF (1996) Anterior and posterior fusion for children with tuberculosis of the spine. Clinical orthopaedics and related research (325):225-231 
22. Cockburn J, Walters WA (1999) Communication between doctors and patients. Curr Obstet Gynaecol $9(1): 34-40$

23. Dyche $L(2007)$ Interpersonal skill in medicine: the essential partner of verbal communication. J Gen Intern Med 22(7):1035-1039.

\section{Figures}

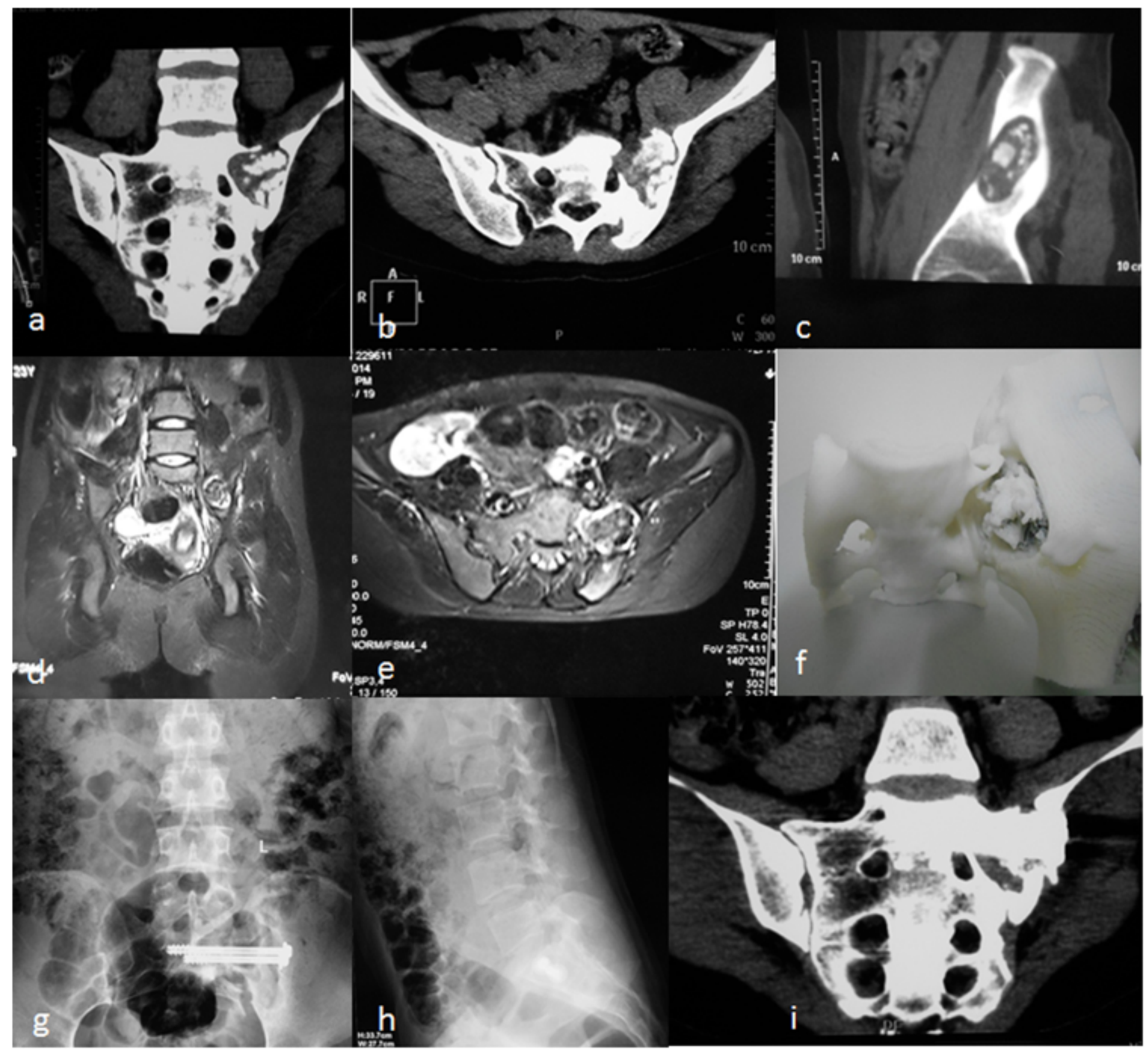

Figure 1 
A 25-year-old female was diagnosed as having sacroiliac joint tuberculosis after an three months history of left buttock. a-e Pre-operative CT and MRI showed that a destructive lesion involving the left sacroiliac joint, and the abscess formation in the left sacroiliac joint. f Surgical simulation on 3D-printed sacroiliac joint model. g-i Post-operative X-ray and CT showed the lesion had healed and radiographs showing good bone fusion. 